\title{
1 Meta-analysis of meta-analyses in plant evolutionary ecology
}

2

3 Maria Clara Castellanos ${ }^{\mathbf{1}}$ and Miguel Verdú ${ }^{\mathbf{2}}$

4 Consejo Superior de Investigaciones Científicas, Centro de Investigaciones sobre

5 Desertificación (CSIC-UV-GV), 46113, Moncada, Valencia, Spain

6

7 11 maclacas@uv.es, ${ }^{2}$ miguel.verdu@uv.es

8

9 Running title: Meta-analysis of meta-analyses in plant evolutionary ecology

10

11 Keywords Biotic and abiotic selective pressures; fitness and non-fitness traits;

12 second-order meta-analysis.

13

14 Total word count (excluding references, tables and figures): 4089 words

15 Word counts for each section:

16 Abstract: 309

17 Introduction: 793

18 Materials and Methods: 1183

19 Results: 421

20 Discussion: 1393

2137 cited references, 1 figure and 1 table

221 online appendix with 51 references 
23 Abstract. After two decades of meta-analyses on plant traits, we can now look for global emergent patterns in plant evolutionary ecology. Hundreds of meta-analyses have focused on the effects of specific selection pressures on plant fitness, and the buildup of such results allows us to ask general questions regarding selection pressures and plant responses, a major focus of evolutionary ecology. Plant traits are affected by both abiotic and biotic factors. For example, biotic pressures like herbivory may affect physiological (i.e. secondary defences) and reproductive (i.e. seed predation) traits. Similarly, abiotic pressures such as increased $\mathrm{CO}_{2}$ may affect both plant physiology and reproduction. We tested whether biotic or abiotic selective pressures are more important for plant traits, and if the strength of the response to those pressures depends on the plant trait studied by meta-analyzing published meta-analyses on plant responses. We classify meta-analyses according to the type of response variable studied (fitness and non-fitness traits) and the type of selective pressure examined (biotic or abiotic). Our database showed biases in the meta-analysis literature, for example that the majority of studies are focused on non-fitness traits, i.e. on traits that are not directly related to reproduction or survival, and furthermore, on non-fitness traits under abiotic selection pressures. The meta-meta-analysis showed that the strength of responses to selection depends on the nature of selection (stronger for biotic than for abiotic factors) but, unexpectedly, not on the type of trait under study as previously found. The stronger responses to biotic factors can be explained if biotic selection is more variable in space and time, driven by interactions with other organisms. The relative importance of biotic 44 versus abiotic factors on plant traits has been little studied in the past, and would benefit from more studies and reviews that fill the under-represented combinations of selective pressures and plant traits (i.e. abiotic factors on fitness traits). 
49 After two decades of meta-analyses on plant traits, beginning with the introduction of the technique in the field of ecology in the early 1990's (see Gurevitch et al. 2001), it is

51 timely to look for global emerging patterns in the literature. In the field of plant evolutionary ecology specifically, hundreds of meta-analytical studies have focused on the effects of specific selection pressures on a single or a few plant fitness components. As a result, generalizations can be made, for example on how increased $\mathrm{N}$ availability or increased herbivore activity can affect plant growth or reproductive output and how such effects hold across experiments and plant species. The buildup of meta-analytic results, however, allows us to go further and ask more general questions regarding selection pressures and plant responses, i.e. the types of questions on patterns of selection that are a major focus of modern evolutionary ecology. Key questions that can be explored are whether biotic or abiotic selective pressures are more important for

61 plants, and if the strength of the response to those pressures depends on the plant trait 62 studied.

Natural selection is an important force behind phenotypic differentiation across a wide range of plant traits (Kingsolver et al. 2001; Rieseberg et al. 2002). However, not all traits are expected to be targeted by selection in the same way. For example, traits closely related to fitness, such as life history traits, are expected to experience stronger

67 selection than other types of traits (Merilä and Sheldon 1999). Tests of this idea have come to different conclusions, depending on the methodological approach. Kingsolver et al. (2001) compared selection gradients and differentials measured in wild populations across different types of traits, and found that morphological traits were subject to stronger selection than life history traits. Rieseberg et al. (2002) on the other 
hand, compared the signature of selection with a more "historical" approach, using the direction of effects of quantitative trait loci (the QTL sign test), and found evidence of stronger and more consistent selection on life history than on morphological characters. Contrasting results are not necessarily surprising because selective pressures are expected to affect plant performance in complex ways (Bell 2010). For example, the strength, form and direction of selection can vary in time (Grant and Grant 2002; Siepielski et al. 2009; Kingsolver and Diamond 2011) and space (Linhart and Grant 1996; Schluter 2000; Herrera et al. 2006) but see also Morrisey and Hadfield (2012). In addition, the type of selection pressure, whether biotic or abiotic, could also exert different responses from plant traits. Biotic selective pressures depend on the interactions with other organisms, such as predators or mutualists, whose distributions and densities can vary rapidly and unpredictably and can therefore be expected to be less consistent in strength, space and time (Linhart and Grant 1996; Thompson 2005). Plant responses to biotic pressures could be then expected to be weaker and less consistent across species and populations than to abiotic pressures. However, a recent study suggests that biotically-selected traits are governed by fewer genes with a large effect, which could allow populations to move faster among variable peaks in an adaptive landscape (Louthan and Kay 2011). Although previous studies have tested for differences between measures of selection on fitness traits compared to other types of traits, to our knowledge no studies have specifically explored the potential differences in selection when the pressures are biotic or abiotic.

We assembled here a database of diverse meta-analyses that allows us to simultaneously test for the strength of the effects of biotic and abiotic selection pressures and the responses of different types of traits (fitness versus non-fitness). We also tested for the interaction between them, which could reveal differential effects of 
97 biotic or abiotic factors on different types of traits. We address these questions quantitatively by performing a meta-analysis of published meta-analyses, or a secondorder meta-analysis, an approach that has been little used in ecology so far, but is

100 already common practice in the medical sciences (usually referred to as "umbrella

101 reviews" when various reviews are compared in narrative form, or "multiple treatment

102 meta-analysis" when multiple meta-analytic results are compared under specific

103 models; Caldwell et al. 2010; Ioannidis 2009). Specifically, we compare, with meta-

104 analytical techniques, a) the global effect sizes of meta-analyses of biotic versus abiotic

105 selection pressures, b) the global effect sizes of fitness versus non-fitness response

106 traits, and c) the interaction between them. Note that we are not dealing with data on

107 selection gradients or differentials (as defined by Lande and Arnold 1983), but with

108 studies that control or measure the selective factors and record their effect on plant

109 traits. In addition, we use our database to describe patterns in the published meta-

110 analysis literature on plant evolutionary ecology and detect potential biases towards

111 certain types of reviews.

112

\section{Materials and methods}

115 Clarification of the terminology used in the remaining of the article follows. The data

116 base used in our qualitative and quantitative analyses is composed of meta-analyses

117 mean effect sizes extracted from publications that may or may not include more than

118 one meta-analysis. Each meta-analysis in turn included original case studies. Data

119 points in our second-order meta-analysis are meta-analyses mean effect sizes and not

120 the original case studies. Methods are detailed below. 
We compiled the data set of published meta-analyses on plant traits by performing

122 a literature search in the Web of Science with topic keywords "meta-analysis and plant"

123 (as of September 2011). We purged down the initial list of around 440 publications to

124 include meta-analyses that met the following requisites. a) Studies had to perform a

125 formal meta-analysis, that is, a comparison of weighted effect sizes across data sets. b)

126 Meta-analyses were revisions of the published literature designed to extract general

127 patterns. This excludes studies that used formal meta-analytical techniques to compare

128 various sets of original data. c) We excluded meta-analyses performed exclusively on

129 crop species under agricultural conditions, because a long history of artificial selection

130 might affect current response to selective pressures. d) We included only meta-analyses

131 focused on plant traits that can be measured in individuals. Community level (e.g.

132 species richness) or ecosystem level traits (e.g. litter decomposition) were not

133 considered. d) We also excluded allometric meta-analyses that were purely

134 morphological (e.g. trunk diameter vs. leaf area), when they had no clear evolutionary

135 implications.

136 We classified the remaining meta-analyses according to the type of response

137 variable studied (growth, physiology, reproduction or survival) and the type of selective

138 pressure examined (biotic or abiotic). Response variables were in turn grouped as

139 fitness variables (reproduction and survival) or non-fitness (physiological and growth

140 traits). This division might not seem straightforward, as it can be argued that growth or

141 development are fitness components as well. Our rationale follows that of Merilä and

142 Sheldon (1999), which assumes that reproductive traits and survival are more closely

143 related to fitness itself than other traits.

144 From each meta-analysis we extracted global effect sizes and their associated

145 sample sizes and sampling error variances to use them as weights. Sampling error 
146 variance is the square of the standard error, but these estimates are seldom reported in

147 the literature. Instead, 95\% confidence interval of the effect size is usually provided and

148 half the width of the $95 \%$ CI divided by 1.96 is a good approximation to the standard

149 error. We did not include partial effect sizes (predictor factors) that subdivide data sets

150 already used to calculate a global effect (e.g. subdividing data sets to test the effect of

151 ant mutualisms on herbivory in shrubs versus herbs, Chamberlain and Holland 2009), to

152 avoid pseudoreplication. When several global effect sizes were provided by the same

153 publication to test separate response variable types (e.g. physiological, reproduction,

154 etc), we included all of them. For example, mutualism effects on growth and

155 reproduction of target plants were studied independently by Trager et al. (2010) and

156 therefore we included two global effects from this publication. Furthermore, if the

157 original meta-analysis mixed the types of response variables we were interested in, we

158 recalculated a global effect size for each variable type if the original data set was

159 available. For example, Bailey et al. (2009) reported effects of introgression on a

160 mixture of physiological, morphological, and reproductive response variables in

161 Populus. We recalculated global effect sizes for growth and physiological response

162 variables separately from their supplementary data set.

163 For our final second-order meta-analysis, we needed to transform individual meta-

164 analyses' effect sizes to a common metric. However, the most common effect metric

165 used in ecological studies, the log of the response ratio (lnRR) cannot be transformed

166 into other metrics in a straightforward way (M. Lejaunesse, pers. com.). We therefore

167 limited our quantitative analysis to meta-analyses reporting $\ln R R$ and closely related

168 metrics (e.g. percentage of change) and excluded those reporting metrics based on

169 standarized mean differences (i.e. Hedges $d$ ) or correlation coefficients. Because the

$170 \ln R R=\ln \left(X^{E}\right)-\ln \left(X^{C}\right)$, i.e. the ratio of the outcome of an experimental group to that of 
171 a control group, our database for the quantitative analysis is composed mostly of metaanalyses of controlled experimental studies, but not exclusively, because some also include original case studies using natural variation (e.g. Chamberlain and Holland 2009; Trager et al. 2010).

There was no significant correlation between effect size and sample size $(r=0.02$, $\mathrm{df}=137, \mathrm{P}=0.81$ ), suggesting against the biased publication of high effect sizes.

Statistical analyses

Because we were only interested in the strength of plant trait responses to selective

181 pressures, the sign of the effect sizes was not informative in our analysis. We therefore

182 used the absolute values of effect sizes ( $\operatorname{lnRR})$ to run Bayesian meta-analyses as explained below. Using the absolute values could introduce an upward bias when estimated effect sizes are non-significantly different from zero (Hereford et al. 2004). However, we do not expect this to affect our comparisons, because around $80 \%$ of the reported meta-analyses were significant. In addition, we are not testing for significance in effect sizes, but rather for differences in their strength.

We first calculated an index of heterogeneity among meta-analyses $\left(\mathrm{I}^{2}\right.$; Higgins and

189 Thompson 2002) using the MCMCglmm R package as suggested by Nakagawa and

190 Santos (2012). Values of $\mathrm{I}^{2}$ around $25 \%, 50 \%$ and $75 \%$ reflect small, medium and large

191 heterogeneity (Higgins et al 2003). For the second-order meta-analysis, we fitted

192 Generalized Linear Mixed Models using Markov chain Monte Carlo techniques with the 193 help of the MCMCglmm package for R (Hadfield 2010). The effect size was the 194 dependent variable in the model, and two types of weights were used: i) sample size and 195 ii) inverse of the sampling error variance. Both weighting strategies have been used in 
196 social sciences (Hunter and Schmidt 2004 and references therein) as well as in ecology

197 (eg., van Groenigen et al 2011). Comparisons of the performance of both methods can

198 be found in Marín-Martínez and Sánchez-Meca (2010) and in Lajeunesse and Forbes

199 (2003).Weights passed to the mev argument of MCMCglmm (Hadfield and Nakagawa

200 2010). We ran 13000 MCMC iterations with a burn-in period of 3000 iterations and

201 convergence of the chain was tested by means of an autocorrelation statistic. The priors

202 used were $n u=0$ and $\mathrm{V}=\mathrm{I}^{*} 1 \mathrm{e}+10$, where $\mathrm{I}$ is an identity matrix of appropriate dimension.

203 The type of selective variable (biotic and abiotic) and the type of response variable

204 (fitness and non-fitness) were included as predictors in the MCMCglmm model,

205 including an interaction. Although separate global effect sizes could come from the

206 same publications, we decided against using the publication as a random grouping

207 factor in the model. This is because 1) separate meta-analyses reported in the same

208 publications are not necessarily non-independent, because they are derived from

209 different sets of original study cases, and 2) publications deal with only one of the

210 selective variable types (biotic or abiotic), so that including it as a random factor would

211 remove important variance from the main predictors unintentionally. The effect of

212 predictors was estimated by calculating the $95 \%$ credible interval of their posterior

213 distribution (Nakagawa and Cuthill 2007).

214

\section{Results}

217 General patterns in the literature

219 Our final data set included 196 meta-analyses based on more than 17800 original study 220 cases, reported in 51 publications (Table 1 and appendix). This sample reflects a bias in 
221 the literature towards meta-analyses of non-fitness traits (154 versus 42 involving

222 fitness responses), and particularly towards those of non-fitness traits under abiotic

223 selection (102 studies). In contrast, only 9 meta-analyses in our data base dealt with

224 biotic characters under abiotic selective pressures.

225 Most abiotic selective pressures were climatic variables (111 vs. only 3 related to

226 disturbance). Among the climatic variables, there is a majority of meta-analyses dealing

227 with responses to elevated $\mathrm{CO}_{2}$ (50 meta-analyses) and exposure to UV-B radiation

228 (25). Biotic pressures are all related to interactions, spanning from ant-plant

229 mutualisms (10 meta-analyses), to herbivory (19), interactions with plant neighbors

230 (11), and less often with plant-microbial interactions, pollinators, etc.

231 Finally, fitness responses are most often some measurement of reproductive output

232 (37 of 42 studies), while survival is the response variable in only 5 studies. In contrast,

233 within non-fitness variables there is a balance between growth and physiological

234 responses (77 each).

235

236 Quantitative analysis

237

238 As explained above, we limit our quantitative analysis to the subset of meta-analyses in

239 our database reporting $\ln R R$ as the effect size $(N=139$ meta-analyses in 30 publications.

240 Sampling error variance could only be obtained from 134 meta-analyses, see appendix).

241 We detected a large value of heterogeneity among meta-analyses $\left(\mathrm{I}^{2}=99.6 \%\right.$; $[99.5$,

242 99.7]), which justified using predictors. Results were very similar for both weighting -

243 sample size and variance- procedures. We found no significant interaction between the

244 type of selective variable (biotic and abiotic) and the type of response variable (fitness

245 and non-fitness) in their effect on effect sizes (posterior mean estimate $=-0.010,95 \%$ 
246 CI [-0.251 to 0.208$]$ for sample size weighted and $-0.043[-0.197,0.079]$ for variance

247 weighted models). We therefore tested for the main effects of the two variables in a 248 model without interaction. It showed no significant differences in effect sizes between

249 fitness and non-fitness response variables $(-0.059,[-0.172,0.044]$ for sample size

250 weighted and $-0.014[-0.086,0.051]$ for variance weighted models). However, there was

251 a significant effect of the type of selective variable analyzed, because biotic variables

252 elicit higher responses than abiotic ones $(0.188[0.104,0.273]$ for sample size weighted

253 and $0.177[0.120,0.234])$. Raw mean effect sizes and their standard errors are shown

254 in Fig. 1. These results are unchanged if we include response variables as physiology,

255 growth, or reproduction traits instead of grouping them as fitness or non-fitness. 
258 Our review of the meta-analytical literature of selection pressures on plants showed, on

259 the one hand, that the majority of meta-analyses are studies of non-fitness traits and

260 mostly on a few abiotic selection pressures such as increased $\mathrm{CO}_{2}$ concentrations. On

261 the other hand, these biases did not prevent a quantitative comparison of the effects of

262 different selective pressures, which showed that the strength of responses to selection

263 depends on the nature of selection (biotic versus abiotic factors) but, unexpectedly, not

264 on the type of trait under selection. We discuss these results below.

266 Trends in the meta-analysis literature

268 Biases in our data base allowed us to detect biases in the meta-analysis literature. The majority of review studies are focused on non-fitness traits, i.e. on traits that are not

270 directly related to reproduction or survival, and furthermore, on non-fitness traits under

271 abiotic selection pressures. Certainly measuring a plant's reproductive output might be 272 more difficult than measuring a morphological or physiological character and this can 273 be one of the reasons for the unbalanced number of reviews. We suspect there is also a 274 tradition of studying plant reproductive responses in a biotic context, and physiological 275 and growth traits as influenced by abiotic environments (see Geber and Griffen 2003). 276 These trends are reinforced by the recent boom of climate change studies, as reflected 277 by the high number of $\mathrm{CO}_{2}$ and UV radiation papers. The differential number of metaanalyses might then reflect a general bias in the plant literature. Louthan \& Kay (2011),

279 for example, also detected a bias towards abiotic-selected traits in a review of plant 280 QTL mapping studies. 
282 Strength of biotic and abiotic selection on fitness and non-fitness traits

283

284 Our approach to comparing the strength of selection on different types of traits differs

285 from other review papers (Kingsolver et al. 2001, Rieseberg et al. 2002, Geber and

286 Griffen 2003) in that we compare the results of multiple meta-analyses in a global,

287 second order meta-analysis that includes thousands of results published in the literature.

288 In addition, we do not focus on phenotypic selection as those articles, but on studies that

289 control biotic or abiotic environmental variation and measure the resulting fitness and

290 non-fitness responses. Because phenotypic selection studies do not formally measure

291 environmental variation, such studies would not be appropriate to test our hypothesis.

292 Still, we can compare our results on response variables to theirs. As opposed to those

293 previous findings, we did not detect differences in the strength of responses to selection

294 among different types of traits, either fitness or non-fitness. In contrast, when we looked

295 for differences in the responses to selection elicited by biotic versus abiotic traits, we

296 found a clear signal. Biotic-driven selection leads to stronger selection on traits in

297 general when compared to abiotic selection pressures, at least for plants. It is possible

298 that the biotic-abiotic comparison absorbs the differences between fitness and non-

299 fitness traits detected in previous studies, as both variables are collinear in our database

300 because of the biases described above.

301 The differential responses to biotic versus abiotic is a question that had been

302 basically unexplored. The main exception is the recent study by with Louthan and Kay

303 (2011), who compiled mapping studies on plant traits and compared the direction and

304 effect sizes of QTLs controlling biotic and abiotic-selected traits. Because they were not

305 dealing with selection studies directly, but rather with the consequences of selection on 
306 the genetic architecture of traits, they classified traits a-priori as putatively abiotic- or

307 biotic-selected. Our study is the first that can confidently assign studies to the type of

308 selection pressure. Our reviewed studies report more immediate responses and the

309 results are therefore less historical than a QTL comparison, but in spite of the difference

310 in approaches, the two studies found consistent results. Louthan and Kay (2011) found

311 QTL's of larger effect associated with biotic-selected traits, and we found stronger

312 observed responses of traits under biotic pressures. Both results are expected for traits

313 that are under variable selective pressures, as can be the case for biotic selective agents.

314 Biotic agents and interactions can vary strongly in space and time (Thompson 2005 and

315 references therein), and consequently produce complex selective landscapes with

316 multiple peaks or peaks that in turn shift in time and space. Such selective scenario can

317 produce phenotypic responses that are stronger than under more subtle abiotic changes,

318 and in turn select for QTLs of major effects.

319 To further explore the relative importance of biotic versus abiotic factors on plant

320 character evolution it is clear that a higher diversity of studies would be very useful. In

321 particular, case studies and meta-analyses in the under-represented categories (fitness

322 traits under abiotic selection and non-fitness traits under biotic selection) would be very

323 valuable. In addition, fully factorial case studies on the effects of biotic and abiotic

324 pressures on both fitness and non-fitness traits in individual species are scant but

325 potentially very informative.

326

327 Guide for future meta-analyses of meta-analysis

328

329 The broad use of formal meta-analytical techniques in plant ecology has undoubtedly

330 contributed to our capacity for summarizing and extracting general results, based on the 
331 strength of combining many varied individual studies. We here take the next step of

332 combining effect sizes of meta-analyses on diverse plant systems and traits in a second

333 order meta-analysis. This approach is already frequently used in the health sciences,

334 particularly to answer clinical questions, where for example different treatments for the

335 same disease need to be compared but results are reported in independent reviews

336 (Ioannidis 2009; Becker and Oxman 2011). Multiple-treatment meta-analysis is used to

337 formally compare meta-analytic results in a network approach that incorporates direct

338 and indirect comparisons of clinical treatments (Hasselblad, 1998; Caldwell et al. 2010).

339 Our analysis is a simplified version of such models.

340 One advantage of the approach of meta-analyzing meta-analyses is that it allows a

341 high level of generalization using a very large number of individual case results already

342 summarized in meta-analyses (in our case, more than 17800) that would be very

343 impractical to attempt with the original studies. Most meta-analyses, except perhaps the

344 most recent ones, do not list each individual study case included and their associated

345 effect size, sample size and variance, all required for a new meta-analysis based on the

346 original studies. In a recent article that used published meta-analyses to find groups of

347 papers on specific topics and extract individual study information (Barto and Rillig

348 2011), the authors report that they had to limit their analysis to a small fraction of the

349 available publications, because few report the necessary data for each case study. In our

350 case, using the original data would then imply going back to each case study and

351 repeating the work done by meta-analytical studies. Another advantage of the second-

352 order approach of using published meta-analyses compared to searching for original

353 case studies is that meta-analyses are prepared by expert authors, who identify the

354 relevant questions on each topic and the appropriate case studies to answer them. In a 
355 broad second-order meta-analysis like ours, such level of expertise is left to the original 356 reviews.

357 Nonetheless, some aspects need to be considered carefully before combining

358 review studies in second-order meta-analysis. First, it is possible that the same

359 individual original studies are included in more than one of the meta-analytic

360 publications available on a given topic. Our questions here were so broad and the

361 number of individual studies on different topics so large, that it is unlikely that this form

362 of pseudoreplication has affected our conclusions. Smaller and more focused meta-

363 meta-analyses should probably be more concerned with excluding replicated results.

364 Care should also be taken not to include meta-analyses that were not careful about

365 another possible form of pseudoreplication, i.e, using the same case studies (and same

366 experimental individuals) to conclude on different effects. Second, there are statistical

367 problems with the conversion of effect sizes to a single common metric, as explained in

368 the Methods section. This can be a problem in ecological studies particularly, because a

369 variety of effect sizes are commonly used and in particular response ratios, whose

370 statistical properties have not been fully studied yet. Because of this problem, we had to

371 limit our quantitative analysis to a single family of effect size metrics and exclude many

372 potentially informative meta-analyses. Finally, future second-order meta-analyses

373 addressing evolutionary issues should ideally include phylogenetic-informed effect

374 sizes (Verdú and Traveset 2004), because of the ubiquity of phylogenetic signal in

375 ecological traits (Blomberg et al. 2003).

377 Acknowledgements

378 We thank J. Sánchez-Meca for comments and discussion on the use of meta-analysis of

379 meta-analyses in ecology and other disciplines, and three reviewers for constructive 
380 comments on an earlier version of the manuscript. S. Nakagawa helped with the

381 statistical analyses. This work was developed under the framework of projects

382 VAMPIRO (CGL2008-05289-C02-01) and the European LinkTree project

383 (BiodivERsA, EUI2008-03713). MCC was supported by a JAE-Doc CSIC scholarship. 


\section{References}

385 Bailey JK, Schweitzer JA, Úbeda F, Koricheva J, LeRoy CJ, Madritch MD, Rehill BJ, 386 Bangert RK, Fischer DG, Allan GJ, Whitham TG (2009) From genes to ecosystems: 387 a synthesis of the effects of plant genetic factors across levels of organization. Philos 388 T Roy Soc B 364 (1523):1607-1616

389 Barto EK, Rillig MC (2011) Dissemination biases in ecology: effect sizes matter more 390 than quality. Oikos DOI: 10.1111/j.1600-0706.2011.19401.x

391 Becker LA, Oxman AD ( 2011) Chapter 22: Overviews of reviews. In: Higgins JPT, 392 Green S (eds) Cochrane Handbook for Systematic Reviews of Interventions Version 393 5.1.0 (updated March 2011). The Cochrane Collaboration. Available from $394 \quad$ www.cochrane-handbook.org

395 Bell G (2010) Fluctuating selection: the perpetual renewal of adaptation in variable 396 environments. Phil Trans R Soc B 365 (1537):87-97

397 Blomberg SP, Garland T, Ives AR (2003) Testing for phylogenetic signal in 398 comparative data: behavioral traits are more labile. Evolution 57:717-745

399 Caldwell DM, Welton NJ, Ades AE (2010) Mixed treatment comparison analysis

400 provides internally coherent treatment effect estimates based on overviews of

401 reviews and can reveal inconsistency. J Clin Epidemiol 63 (8):875-882

402 Chamberlain SA, Holland JN (2009) Quantitative synthesis of context dependency in 403 ant-plant protection mutualisms. Ecology 90 (9):2384-2392

404 Geber MA, Griffen LR (2003) Inheritance and natural selection on functional traits. Int 405 J Plant Sci 164 (3 Suppl.):S21-S42

406 Grant PR, Grant BR (2002) Unpredictable evolution in a 30-year study of Darwin's 407 finches. Science 296:707-711 
408 Groenigen, KJ , Osenberg CW, Hungate BA. 2011. Increased soil emissions of potent

409 greenhouse gases under increased atmospheric CO2. Nature 475: 214-216.

410 Gurevitch J, Curtis PS, Jones MH (2001) Meta-analysis in Ecology. Advances in

$411 \quad$ Ecological Research 32:199-247

412 Hadfield JD (2010) MCMC methods for multi-response generalised linear mixed

413 models: the MCMCglmm R package. J Stat Sci 33 (2):1

414 Hadfield JD, Nakagawa S (2010) General quantitative genetic methods for comparative

415 biology: Phylogenies, taxonomies and multi-trait models for continuous and

416 categorical characters. J Evol Biol 23 (3):494-508

417 Morrissey M, Hadfield J (2012) Directional selection in temporally replicated studies is 418 remarkably consistent. Evolution DOI: 10.1111/j.1558- 5646.2011.01444.x.

419 Hasselblad V (1998) Meta-analysis of multitreatment studies. Med Decis Making 18

$420 \quad(1): 37-43$

421 Hereford J, Hansen TF, Houle D (2004) Comparing strengths of directional selection:

422 how strong is strong? Evolution 58 (10):2133-2143

423 Herrera CM, Castellanos MC, Medrano M (2006) Geographical context of floral

424 evolution: towards an improved research programme in floral diversification. In:

425 Harder LD, Barrett SCH (eds) Ecology and Evolution of Flowers. Oxford University

426 Press, New York, pp178-294

427 Higgins JPT, Thompson SG (2002) Quantifying heterogeneity in a meta-analysis.

$428 \quad$ Statist Med $21(11): 1539-1558$

429 Higgins JPT, Thompson SG, Deeks JJ, Altman DG (2003) Measuring inconsistency in $430 \quad$ meta-analyses. British Medical Journal 327:557-560

431 Hunter, J. E. \& Schmidt, F. L. (2004). Methods of meta-analysis (2nd ed.). Thousand 432 Oaks, CA: Sage. 
434 Ioannidis JP (2009) Integration of evidence from multiple meta-analyses: a primer on 435 umbrella reviews, treatment networks and multiple treatments meta-analyses. CMAJ $436181(8): 488-493$

437 Kingsolver JG, Diamond SE (2011) Phenotypic selection in natural populations: what $438 \quad$ limits directional selection? Am Nat 177 (3):346-357

439 Kingsolver JG, Hoekstra HE, Hoekstra JM, Berrigan D, Vignieri SN, Hill CE, Hoang 440 A, Gibert P, Beerli P (2001) The strength of phenotypic selection in natural $441 \quad$ populations. Am Nat 157 (3):245-261

442 Lajeunesse, M.J. and Forbes, M.R. (2003) Variable reporting and quantitative reviews: 443 a comparison of three meta-analytical techniques. Ecology Letters 6, 448-454

444 Lande R, Arnold SJ (1983) The measurement of selection on correlated characters. $445 \quad$ Evolution 37:1210-1226

446 Linhart YB, Grant MC (1996) Evolutionary significance of local genetic differentiation 447 in plants. Annu Rev Ecol Syst 27:237-277

448 Louthan AM, Kay KM (2011) Comparing the adaptive landscape across trait types: 449 larger QTL effect size in traits under biotic selection. BMC Evol Biol 11:60 450 Marín-Martínez, F. and Sánchez-Meca, J. (2010). Weighting by inverse variance or by 451 sample size in random-effects meta-analysis. Educational and Psychological 452 Measurement 70, 56-73.

453 Merilä J, Sheldon BC (1999) Genetic architecture of fitness and nonfitness traits:

454 empirical patterns and development of ideas. Heredity 83:103-109

455 Nakagawa S, Cuthill IC (2007) Effect size, confidence interval and statistical 456 significance: a practical guide for biologists. Biol Rev 82 (4):591-605 
457 Nakagawa S, Santos ESA (2012). Methodological issues and advances in biological

458 meta-analysis. Evol. Ecol DOI 10.1007/s10682-012-9555-5.

459 Rieseberg LH, Widmer A, Arntz AM, Burke JM (2002) Directional selection is the

460 primary cause of phenotypic diversification. Proc Natl Acad Sci USA 99 (19):12242-

$461 \quad 12245$

462 Schluter D (2000) The ecology of adaptive radiation. Oxford series in Ecology and

463 Evolution. Oxford University Press, New York

464 Siepielski AM, DiBattista JD, Carlson SM (2009) It's about time: the temporal

465 dynamics of phenotypic selection in the wild. Ecol Lett 12 (11):1261-1276

466 Thompson JN (2005) The Geographic Mosaic of Coevolution. University of Chicago

467 Press, Chicago

468 Trager MD, Bhotika S, Hostetler JA, Andrade GV, Rodriguez-Cabal MA, McKeon CS,

469 Osenberg CW, Bolker BM (2010) Benefits for plants in ant-plant protective

470 mutualisms: a meta-analysis. PLoS One 5 (12):e14308

471 Verdú M, Traveset A (2004) Bridging meta-analysis and the comparative method: A

472 test of seed size effect on germination after frugivores' gut passage. Oecologia 138:

$473 \quad 414-418$. 


\section{$474 \quad$ Figure legends}

475

476 Figure 1. Raw mean and 1 standard error of effect sizes (lnRR) for meta-analyses

477 classified according to the type of selective variable (biotic and abiotic) and the type of

478 response variable (fitness and non-fitness). Sample sizes for each group are included. 
479 Table 1. Number of meta-analyses in each category of selective pressures and trait

480 response types included in this revision. Details and references are in appendix 1.

481

\begin{tabular}{|c|c|c|}
\hline $\begin{array}{l}\text { Selective } \\
\text { pressure }\end{array}$ & $\begin{array}{l}\text { Response trait } \\
\text { type }\end{array}$ & $\begin{array}{l}\text { Meta-analyses } \\
\text { in this study }\end{array}$ \\
\hline \multirow[t]{6}{*}{ Biotic } & Fitness & \\
\hline & reproduction & 28 \\
\hline & survival & 5 \\
\hline & Non-fitness & \\
\hline & physiology & 14 \\
\hline & growth & 38 \\
\hline \multirow[t]{6}{*}{ Abiotic } & Fitness & \\
\hline & reproduction & 9 \\
\hline & survival & 0 \\
\hline & Non-fitness & \\
\hline & physiology & 63 \\
\hline & growth & 39 \\
\hline
\end{tabular}

482 


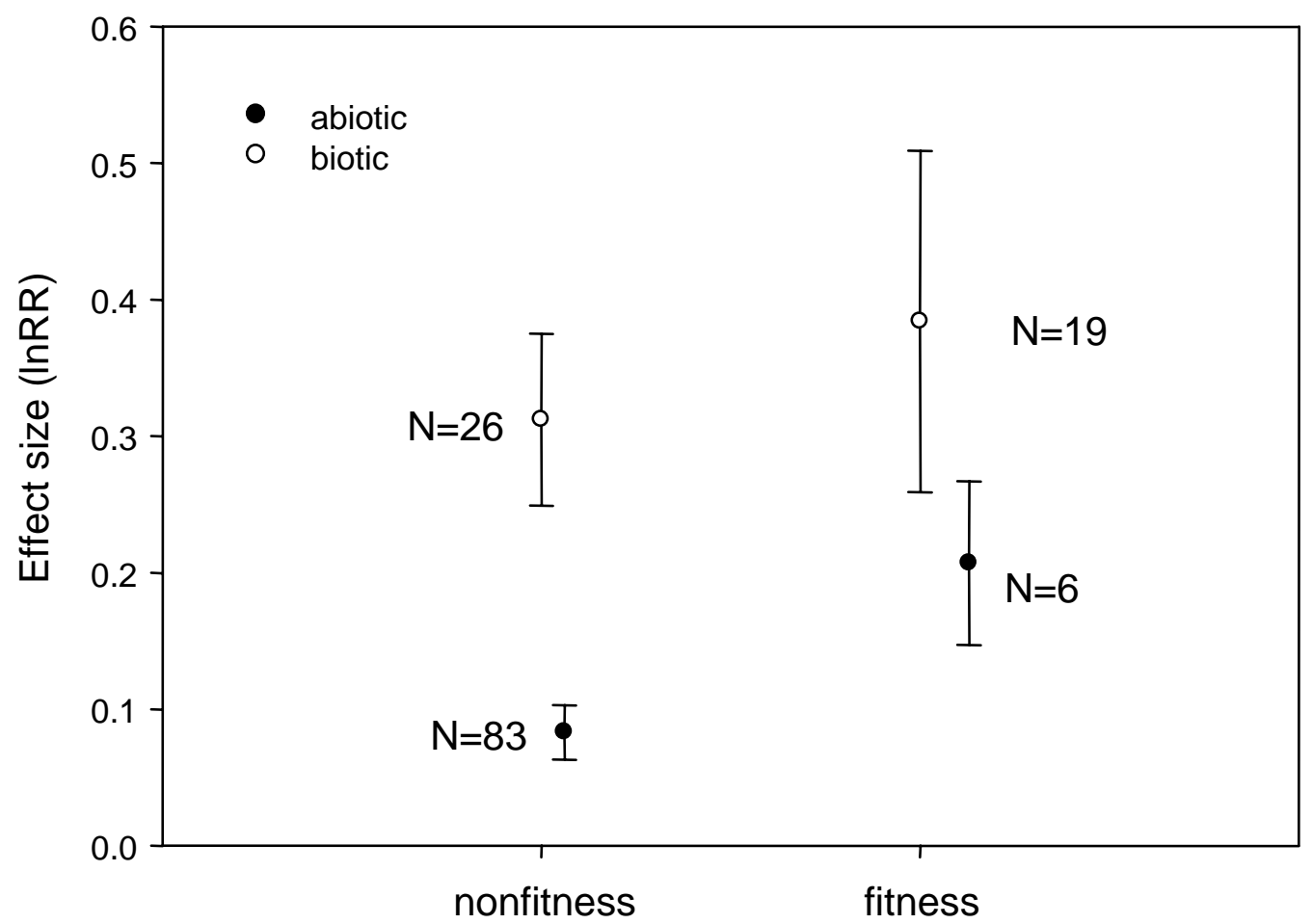

